\title{
The Multispectral Method: Progress and Prospects
}

\author{
Malcolm Roberts $^{1}$, John C. Bowman ${ }^{1}$, and Bruno Eckhardt ${ }^{2}$ \\ ${ }^{1}$ Dept. of Mathematics, University of Alberta, Edmonton, AB, T6G 2G1, Canada \\ ${ }^{2}$ Fachbereich Physik, Philipps-Universität Marburg, 35032 Marburg, Germany \\ mroberts at math.ualberta.ca
}

\section{Introduction}

Simulations of high Reynolds-number turbulence require a large number of Fourier modes, with no natural scale separation of which we might take advantage. The underlying hyperbolic system of equations is too large to be simulated on any available computer system.

The technique of spectral reduction [1] is a decimation scheme that allows one to simulate large systems on uniformly-coarsened spectral grids. The multispectral method uses a hierarchy of differently-coarsened grids in Fourier space, retaining all large-scale modes while approximating the smaller scales.

\section{The models in question}

Since high-Reynolds number simulations of the Navier-Stokes equations are not currently tenable, we introduce two shell models of turbulence, the DN [3] model,

$$
\frac{d u_{n}}{d t}=i k_{n}\left(a u_{n-1}^{2}-\lambda a u_{n} u_{n+1}+b u_{n-1} u_{n}-\lambda b u_{n+1}^{2}\right)^{*}-\nu k_{n}^{2} u_{n}
$$

and the GOY $[4,5]$ model,

$$
\frac{d u_{n}}{d t}=i k_{n}\left(\alpha u_{n+1} u_{n+2}+\frac{\beta}{\lambda} u_{n-1} u_{n+1}+\frac{\gamma}{\lambda^{2}} u_{n-1} u_{n-2}\right)^{*}-\nu k_{n}^{2} u_{n}
$$

where ${ }^{*}$ denotes complex conjugation. These are heuristic models that mimic the form and many statistical properties of the spectral Navier-Stokes equations. Because of these features, they are excellent test beds for theories about turbulence.

The modal amplitude $u_{n}$ associated with the geometrically spaced wavenumber $k_{n}=k_{0} \lambda^{n}$ represents a characteristic complex amplitude of the modes 
$\mathbf{u}(\mathbf{k})$ for $|\mathbf{k}| \in\left[k_{n}, k_{n+1}\right]$. That is, $u_{n}$ represents a typical velocity for a threedimensional shell with inner radius $k_{n}$ and outer radius $k_{n+1}$, as shown in Fig. 1. The combination of averaging and geometric spacing allows one to reach high wavenumbers, and hence very high Reynolds numbers, with a small number of modes, as shown in Fig. 2.

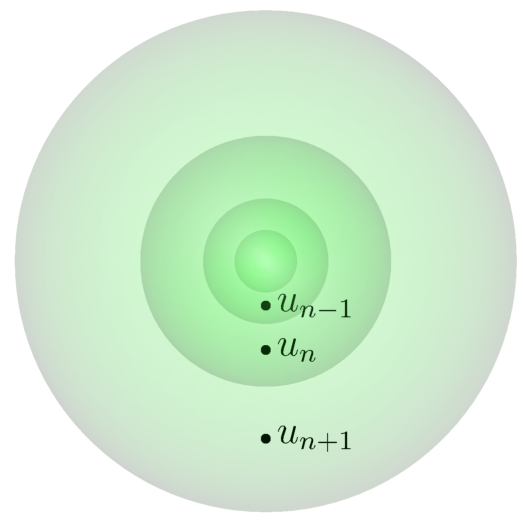

Fig. 1. Spectral domain for mode $u_{n}$.

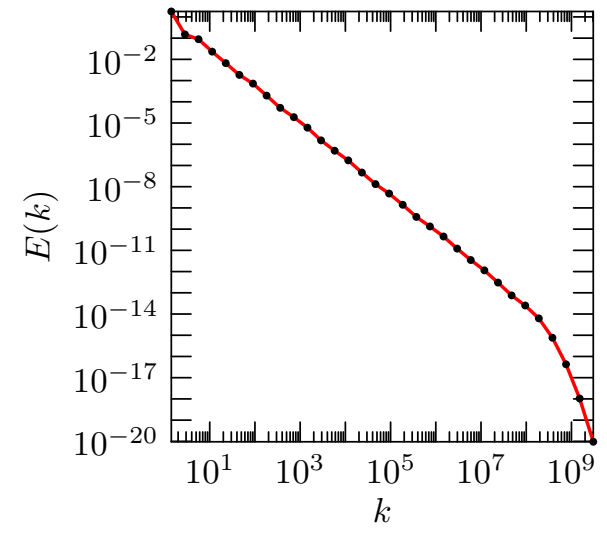

Fig. 2. Energy spectrum of shell-model turbulence.

\section{Spectral Reduction}

Spectral reduction allows one to take two modes, say $u_{2 n}$ and $u_{2 n+1}$, and replace them with an average of the two full-resolution modes $u_{2 n}$ and $u_{2 n+1}$ :

$$
u_{n, 1}=\frac{u_{2 n}+u_{2 n+1}}{2}
$$

This uniform coarsening allows one to simulate a binned energy spectrum using half as many modes. Spectral reduction can be applied iteratively to the system until there are too few remaining independent modes to capture the energy injection, inertial-range transfer, and dissipation.

An equation for $\frac{d}{d t} u_{n, 1}$ follows on taking the derivative of equation (3) and substituting either equation (1) or (2), depending on whether one is simulating the DN or GOY models, respectively. The resulting system will conserve the binned energy $E_{1}=\frac{1}{2} \sum 2\left|u_{n, 1}\right|^{2}$. An easy calculation shows that spectral reduction reduces the GOY model to the DN model, which is then a fixed point of the map, with each reduction modifying the parameters $a, b$ and $\nu$ as per equation (4): 


$$
a \rightarrow \frac{a}{2}, \quad b \rightarrow \frac{b}{2} \quad \nu \rightarrow \nu \frac{1+\lambda^{2}}{2}
$$

The energy spectrum of a singly decimated system is shown in Fig. 3.

\section{Interpolation}

The binned mode $u_{n, 1}$ represents two quantities. We can recapture some of this structure by replacing equation (3) with

$$
u_{n, 1}=\frac{u_{2 n}+\sigma_{n}^{*} u_{2 n+1}}{1+\left|\sigma_{n}\right|^{2}}, \quad \sigma_{n}=\frac{u_{2 n+1}}{u_{2 n}} .
$$

The complex quantity $\sigma_{n}$ accounts for the slope of the amplitude across a bin. If $\sigma_{n}$ is constant with respect to time, then the energy $E_{1}=\frac{1}{2} \sum_{n}(1+$ $\left.\left|\sigma_{n}\right|^{2}\right)\left|u_{n, 1}\right|^{2}$ is conserved. This interpolated spectral reduction can also be applied iteratively, with the caveat that energy is conserved only when $\sigma$ is constant during a time step and over each coarse bin. In order to close the system, we let

$$
\sigma_{n}=\sqrt[4]{\left\langle\left|u_{n, 1}\right|^{2}\right\rangle /\left\langle\left|u_{n+1,1}\right|^{2}\right\rangle}
$$

where $\langle\ldots\rangle$ is a windowed time average. The energy spectrum of an interpolated singly decimated system is shown in Fig. 3.

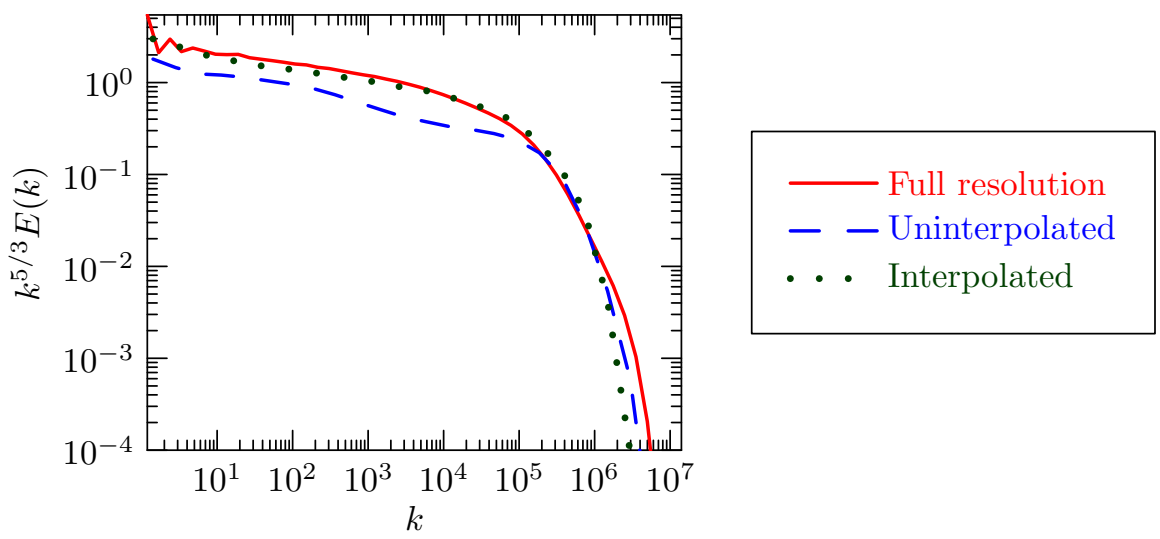

Fig. 3. Rescaled energy spectrum of spectrally reduced shell-model turbulence. 


\section{The Multispectral Method}

The method of spectral reduction allows one to represent a large system on a uniformly coarsened spectral grid. However, the low-wavenumber modes are typically of greater physical importance: one would like to coarsen just the high-wavenumber, small-scale modes in favour of keeping all of the lowwavenumber, large-scale modes. Unfortunately, this modifies the system's Liouville theorem, and the inviscid, unforced energy spectrum is not correctly reproduced.

The multispectral method was designed to alleviate this problem. Since we are unable to decimate a single grid non-uniformly, we must use two grids which have been decimated to different degrees, as shown in Fig. 4.

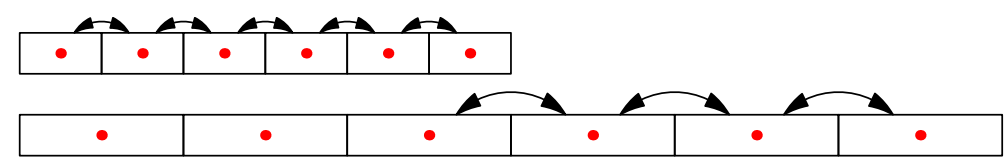

Fig. 4. Schematic diagram for the DN model with the multispectral method showing nonlinear interactions.

To evolve this system forward in time, redundant interactions are first removed. One then evolves the fine grid from time $t$ to $t+d t$ using an explicit integrator. The result of this integration is projected onto the coarse grid, which is then also evolved from from time $t$ to $t+d t$ using a (possibly different) explicit integrator. Finally, the two grids are synchronised by prolonging from the coarse grid to the fine grid.

The resulting system reproduces the essential behaviour of the full-resolution system, keeps all the large-scale modes, but is much less costly to simulate.

\section{References}

1. Bowman, Shadwick, and Morrison, Phys. Rev. Lett. 83, 5491 (1999).

2. J. C. Bowman, C. R. Doering, B. Eckhardt, J. Davoudi, M. Roberts, and Jörg Schumacher, Physica D 218, 1-10 (2006).

3. V. N. Desnyansky and E. A. Novikov, Prikl. Mat. Mekh. 38, 507 (1974)

4. E. B. Gledzer, System of hydrodynamic type admitting two quadratic integrals of motion, Sov. Phys. Dokl., 18, 216-217, (1973)

5. Yamada, Michio and Ohkitani, Koji, Asymptotic formulas for the Lyapunov spectrum of fully developed shell model turbulence, Phys. Rev. E 57, 62576260 (1998) 